\title{
Deficiencia de IgA: análisis clínico
}

\author{
Dres. Roger Lamas S."•, Juan Quintana**, Liliana \\ Rebolledo** y Ricardo Sörensen***
}

En estudios efectuados en diferentes países se ha comprobado que $1 \times 500$ a $1 \times 3.000$ personas aparentemente normales, presentan déficit de IgA. $(1,3,5)$ Los mecanismos genéticos por los cuales se produce esta deficiencia no están bien claros. (4) Se ha descrito su asociación con diversos cuadros clínicos en el niño, siendo los más frecuentemente citados los siguientes: infecciones respiratorias y del tracto digestivo, síndrome de mala absorción, hipersensibilidad (atopia), enfermedades autoinmunes, ataxia-telangiectasia, hiperplasia linfática y tumores, particularmente linfomas, $y$ otras que sería largo enumerar. $(1,3,5,6$, $7,8,9,10$ )

La presente comunicación tiene por objeto mostrar brevemente un grupo de 18 niños controlados en la Unidad de Inmunología del Hospital Calvo Mackenna, en el periodo 19731975, con variadas manifestaciones clinicas, y en los que comprobo disminución de los niveles de IgA.

\section{MATERIAL Y METODO}

Aparte de una anamnesis y examen físico completos, el estudio inmunológico de los

\footnotetext{
- Presentado como Relato Oficial en las V Jornadas Anuales de la Sociedad Chilena de Pediatría, Lo Barnechea, 1975.

**Unidad de Inmunología, Hospital Luis Calvo. Mackenna.

"*"Unidad de Inmunologia, Hospital Luis Calyo Mackenna y Departamento Inmunologia, Instituto Bacteriológico de Chile.
}

pacientes incluyo los siguientes exámenes de laboratorio: Hemograma (neutrófilos-linfocitos); Batería inmunologica (cuantificación de inmunoglobulinas y complemento séricos), con estudio familiar en 3 niños; Tests cutáneos de inmunidad celular: PPD,+ DNCB (Dinitroclorobenceno), antígeno de Monilia; Fenómenos autoinmunes: AAN (anticuerpos antinucleares), FR (factor reumatoide: prueba de látex y Waaler-Rose), y células LE, exámenes que se efectuaron en 6 pacientes.

De acuerdo con los valores de IgA encontrados, se dividió a los niños en 3 grupos:

a) Deficiencia selectiva de IgA: aquellos que tienen menos de $5 \mathrm{mg} \%$ de IgA sérica.

b) Deficiencia parcial de IgA: los que presentan cifras repetidamente inferiores a Ias normales para su edad, según determinaciones efectuadas en nuestro medio.

c) Deficiencias de IgA asociadas a otras inmunodeficiencias: niños con deficit total o parcial de IgA, en los que se comprobó alguna otra deficiencia, ya sea de la inmunidad humoral o celular.

\section{RESULTADOS}

a) Deficiencia selectiva de IgA (Tabla No 1): comprende 3 niñas con artritis reumatoldea juvenil, 2 de las cuales presentan fenómenos autoinmunes positivos, y un preescolar de 5 años, de sexo masculino, con síndrome nefrótico idiopático. 
Tabla N: I

DEFICIENCIA SELECTIVA DE IgA (menos de $5 \mathrm{mg} . \%$ )

\begin{tabular}{|c|c|c|c|c|c|c|c|}
\hline Nombre & Edad & Sexo & $J g M$ & $J_{g} G$ & $J g A$ & Fen. attoinmumes & Diagnóstico \\
\hline A.M. & $8 a$. & Fem. & $\mathbf{N}$ & $\mathbf{N}$ & A & $\begin{array}{l}\text { F.R.-1:60 (Látex) } \\
1: 80(") \\
\text { A.A.N. (+) y (-) } \\
\text { CÉl. L.E. (-) }\end{array}$ & $\begin{array}{l}\text { A.R. juyenil } \\
\text { Infecciones a repeticion }\end{array}$ \\
\hline L. $\mathbf{M}$. & 148. & Fem. & $\mathrm{N}$ & $\mathbf{N}$ & A & $\begin{array}{l}\text { A.A.N. } \\
\text { Cél. L.E. } \stackrel{1: 100}{(-200}\end{array}$ & $\begin{array}{l}\text { A.R. juvenil } \\
\text { Alergia por picaduras } \\
\text { de insectos }\end{array}$ \\
\hline A. $\mathbf{R}$. & $6 a$. & Fem. & $\mathbf{N}$ & $\mathbf{N}$ & A & & Obs. A.R. juvenil \\
\hline J, $\mathbf{G}$. & 3a. & Masc. & $\mathbf{E}$ & $\mathbf{N}$ & $\mathbf{A}$ & & Sindrome nefrótico idiopético \\
\hline
\end{tabular}

$\mathbf{N}=$ Normal $\quad \mathbf{E}=$ Elevado $\quad \mathrm{A}=$ Autsente 
Los exámenes efectuados para evaluar defensas inespecificas e inmunidad celular en todos ellos, fueron normales.

Como patología asociada encontramos infecciones a repetición en una niñe (amigdalitis, faringitis, erisipela prolongada), y alergia por picaduras de insectos en la segunda, sin alcanzar caracteres de gravedad en ninguna de ellas.

En la primera de las mencionadas se encontró además IgA secretora en saliva menor de $1 \mathrm{mg} \%$, IgE presente (test cutáneo), e isoaglutininas normales. Las determinaciones de inmunoglobulinas en sus padres y hermanos fueron normales.

b) Deficiencia parcial de IgA (Tabla No 2): Este es el grupo más numeroso y comprende 10 niüos desde los 5 meses hasta 12 años. Predominan las infecciones respiratorias a repetición (6 niños), 3 niñas escolares presentaban artritis reumatoidea, y una lactante de 6 meses, timectomizada 1 mes antes con diagnóstico de tumor mediastínico y fenómenos compresivos a nivel bronquial y que en la actualidad también presenta infecciones respiratorias altas a repetición, junto a raquitismo resistente a la vitamina $A$.

En 2 enfermos encontramos elevación de IgG, y en uno, de IgM, que interpretamos como debidas a procesos infecciosos concomitantes.

Al igual que en el grupo anterior, cabe destacar la presencia de fenómenos autoinmunes positivos en las 3 niñas con artritis reumatoídea, y en un lactante de 5 meses con infecciones respiratorias a repetición.

c) Deficiencia de IgA asociada a otras inmunodeficiencias (Tabla No 3): Jncluye 4 niños que tienen en común: el presentar cuadros clínicos abigarrados, con numerosas infecciones a repetición, entre las que predominan las respiratorias, digestivas y urinarias. En los casos de deficiencia asociala de la inmunidad celular, se presentan además infecciones por virus diversos, lamblias y hongos.

Corresponden a los casos más severos de nuestra casuística, $y$ en ellos encontramos (salvo en el primero) que su patología los ha llevado a un evidente deterioro de su estado general, explicable al revisar diagnósticos que figuran en la Tabla. A este respecto, cabe consignar que la paciente asignada $M . N$., es la única desnutrida de los tres grupos, y estaba catalogada como tal descle antes del inicio de sus cuadros infecciosos a repetición.

\section{DISCUSION}

Se conoce el papel neutralizante de la IgA frente a los virus y a ciertos antígenos extraños, especialmente proteínas de la alimentación, existiendo actualmente también evidencias de que actuaría contra bacterias a través de la vía alterna de activación del complemento. Además, estimula la fagocitosis por parte de los monocitos. (2)

Según vemos en la Tabla № 4 , la deficiencia de IgA a nivel de secreciones y en el suero, puede condicionar la existencia de patología local o sistemática, la cual se expresaría de diversas maneras, de acuerdo con el tipo de respuesta provocada por la penetración del agente patógeno.

Por ser una inmunoglobulina predominante a nivel de secreciones externas, observamos que su déficit redunda en muchos casos en infecciones de orgainos o sistemas en cuya pared existe síntesis de ella (infecciones respiratorias, digestivas, urinarias).

Hay numerosas publicaciones en relación con los diversos cuadros descritos en asociación con deficiencias de IgA y sus posibles mecanismos patogénicos, $(1,2,5,6,7,8,9$, 10) por lo cual no entraremos en más detalles a este respecto. Solo pretendemos hacer notar ciertos hechos que se desprenden del análisis de los enfermos presentados.

En primer lugar, vemos que de acuerdo con la teoria de Cooper sobre el orden ontogénico y filogérico de desarrollo de las inmunoglobulinas, a saber:

$$
\mathrm{IgM} \longrightarrow \operatorname{IgG} \longrightarrow \mathrm{C} \text { IgA. }
$$

en todos los casos analizados se cumple esta secuencia, ya que encontramos IgA disminui- 
DEFICIENCIA PARCIAL DE IgA

\begin{tabular}{|c|c|c|c|c|c|c|c|}
\hline Nombre & Edad & Sexo & $J g M$ & $\operatorname{IgG}$ & $\operatorname{IgA}$ & Fen, autoinmunes & Diagnóstico \\
\hline G.T. & $5 \mathrm{mb}$. & Masc. & $\mathbf{N}$ & $\mathbf{N}$ & D & A.A.N. $1: 200$ & Inf. respiretorias a repetición \\
\hline M.C.M. & $6 \mathrm{~m}$. & Fem. & $\mathrm{N}$ & $\mathbf{E}$ & D & & $\begin{array}{l}\text { Tímectomizada } \\
\text { Inf. respiratorias a repetición } \\
\text { Raquitismo }\end{array}$ \\
\hline V.S.Z. & $11 \mathrm{~m}$. & Fetn. & $\mathbf{E}$ & $\mathbf{N}$ & D & & $\begin{array}{l}\text { Inf. respiratorias a repetición } \\
\text { Inf. urinaria }\end{array}$ \\
\hline M.O. & $11 \mathrm{~m}$. & Fem. & $\mathbf{N}$ & $\mathbf{E}$ & $\mathbf{D}$ & & Bronquitis obstruct. recidivante \\
\hline P.E. & $15 \mathrm{~m}$. & Mase. & $\mathbf{N}$ & $\mathbf{N}$ & D & & Inf. respiratorias a repetición \\
\hline M.M. & $20 \mathrm{~m}$. & Masc. & $\mathbf{N}$ & $\mathbf{N}$ & $\mathbf{D}$ & & Inf. respiratorias a repetición \\
\hline \multirow{2}{*}{$\begin{array}{l}\text { M.T. } \\
\text { V.S.S. }\end{array}$} & $21 \mathrm{~m}$. & Fem. & $\mathbf{N}$ & $\mathbf{N}$ & $\mathbf{D}$ & & $\begin{array}{l}\text { Inf. respiratorias a repetición } \\
\text { (6 bronconeumonias) }\end{array}$ \\
\hline & $8 a$. & Fem. & $\mathbf{N}$ & $\mathbf{N}$ & $\mathbf{D}$ & A.A.N. 1:100 & A.R. juvenil \\
\hline E.P. & $9 a$. & Fem. & $\mathbf{N}$ & $\mathbf{N}$ & $\mathbf{D}$ & $\begin{array}{l}\text { A.A.N. (-) } \\
\text { F.R.R. }\end{array}$ & $\begin{array}{l}\text { A.R. juvenil } \\
\text { Inf. urinaria }\end{array}$ \\
\hline A.B. & $12 a$ & Fem. & $N$ & $N$ & D & $\begin{array}{l}\text { A.A.N. } 1: 100 \\
\text { W.R. }(-)\end{array}$ & A.R. juvenil \\
\hline
\end{tabular}

$N=$ Normal $\quad E=$ Elevada $\quad D=$ Disminuida

da $\mathrm{o}$ ausente con IgM e IgG normales, y en 2 casos, IgG e IgA disminuidas, con IgM normal. No vemos la situación contraria, es decir, IgM o IgG disminuidas con IgA normal. Asimismo, en los casos que se determino IgA secretora en saliva, la encontramos disminutida, al igual que la IgA sérica. No hemos observado deficiencias de IgA secretora en presencia de IgA sérica normal.

Un hecho inuy llamativo es la frecuente asociación de la deficiencia de IgA con fenbmenos autoinmunes positivos, la que ocurre según algunos autores aproximadamente en un $40 \%$ de los casos. (8) En nuestros niños, de 6 investigados, todos presentaron algún tipo de reacción positiva. De éstos, 5 corresponden a niñas con diagnóstico de Artritis Reumatoídea, 2 de las cuales tuvieron Factor Reumatoídeo positivo, el que es muy raro de encontrar en los casos de AR juvenil no asociada a esta inmunodeficiencia. Por ello, es recomendable investigar fenomenos de autoinmunidad en todos los niños con deficiencia de IgA, y hacer un seguimiento a largo plazo de los casos positivos. 
Tobla Na 3

DEFICIENCIA DE IgA ASOCIADA A OTRAS INMUNODEFICIENCIAS

\begin{tabular}{|c|c|c|c|c|c|c|c|c|c|c|}
\hline \multirow[b]{2}{*}{ Nombre } & \multirow[b]{2}{*}{ Edad } & \multirow[b]{2}{*}{ Sexo } & \multicolumn{2}{|c|}{ Def. inespecificas } & \multicolumn{2}{|c|}{ Inmun. celular } & \multicolumn{3}{|c|}{ Inmun. humoral } & \multirow[b]{2}{*}{ Diagnóstico } \\
\hline & & & Nentrof. & Complem. & Linfo. & Test Cut. & $\operatorname{Ig} M$ & IgG & $I g A$ & \\
\hline MM. & 5a. & Masc. & $\mathbf{N}$ & $\mathbf{N}$ & $\mathbf{N}$ & PPD (+) & $\mathbf{N}$ & D & $\mathbf{A}$ & $\begin{array}{l}\text { Infecciones a repetición } \\
\text { Deficiencia inmunidad } \\
\text { humoral compuesta * }\end{array}$ \\
\hline G.M. & $5 a$. & Masc. & $\mathbf{N}$ & $\mathbf{N}$ & $\mathbf{N}$ & PPD (+) & NoD & $\mathbf{D}$ & $\mathbf{D}$ & $\begin{array}{l}\text { Leucemia aguda linfoblástica } \\
\text { Infecciones a repetición "* } \\
\text { Síndrome de malabsorclón } \\
\text { Hepatitis crónica activa } \\
\text { Deficiencias inmunidad } \\
\text { humoral }\end{array}$ \\
\hline G.P. & $10 a$. & Fem. & $\mathbf{N}$ & $\mathbf{N}$ & $\mathbf{N}$ & $\begin{array}{l}\text { PPD (-) } \\
\text { Monillia (-) } \\
\text { INCB (-) }\end{array}$ & $\mathbf{N}$ & $\mathbf{E}$ & $\mathbf{A}$ & $\begin{array}{l}\text { Infecciones respiratorias } \\
\text { a repeticion } \\
\text { Bronquiectasias bilaterales } \\
\text { Deficiencia inmunidad } \\
\text { celular y Deficiencia IgA }\end{array}$ \\
\hline M.N. & $5 \mathrm{~m}$. & Fem. & $\mathbf{N}$ & $\mathbf{N}$ & $\mathbf{N}$ & $\begin{array}{l}\operatorname{PPD}(-) \\
\text { Monilia (-) } \\
\text { DNCB (-) }\end{array}$ & $\mathbf{N}$ & $\mathbf{N}$ & A & $\begin{array}{l}\text { Infecciones a repetición } \\
\text { (Diarrea - Inf. urinaria- } \\
\text { Otitis media aguda) } \\
\text { Moniliasis }\end{array}$ \\
\hline \multicolumn{11}{|c|}{$\begin{array}{l}\text { "Se encontró además deficiencias do IgA secke tora en saliva y líquido sinusal, ausencia de Isoaglu- } \\
\text { tininas anti-B y test cutíneo para IgE }(- \text {. Ausencia de plasmocitos en folfculos linfoides ade- } \\
\text { noídeos. Un hermano de este paciente presento IgA disminuida en forma persistente. } \\
\text { "*Bronquitis. Infección urinarla - Diarrea - Parotiditis (2) - Varicela - Herpes zóster (2) - } \\
\text { Lambliasis - Balanitis. } \\
\text { **Presentó también ausencla de IgA secretora en saliva. } \\
\text { ***Paciente desnutrida con infecciones recurrentes durante todo el primer afio de vida. }\end{array}$} \\
\hline
\end{tabular}




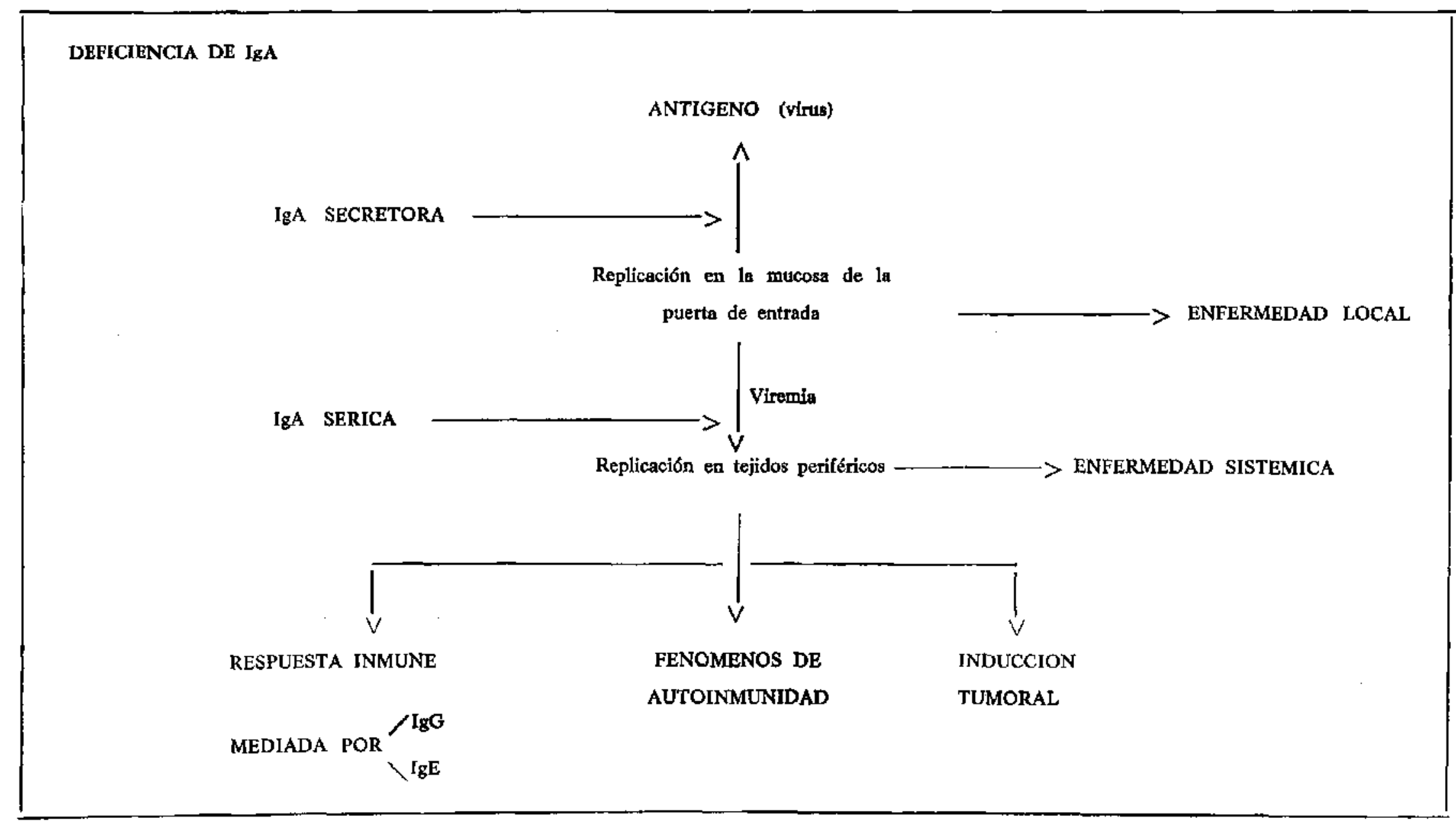

Tabla № 4

Modificado de Tomasi, Th. B., New Eng. J. Med. Vol. 287 N? 10, Sept. 7, 1972. 
Esto tiene su apoyo también en lo informado por algunos autores extranjeros, que muestran series clínicas de adultos con déficit de IgA, en los que predominan las enfermedades autoinmunes.

La evolución de estos niños es varjable, según el tipo de deficiencia que presentan, apreciándose que, salvo excepciones, no es grave en los casos puros, adquitiendo mayor gravedad cuando se asocia a otros déficits de Ja inmunidad humoral o celular.

Aunque no tenemos un seguimiento adecuado de todos ellos, en algunos se aprecia tendencia a la mejoría clínica conjuntamente con la normalización de los niveles de IgA. Este es el caso de EP (Tabla No 2), portadora de una AR poliarticular con FR ( + ), la cual está actualmente asintomática y con IgA normal.

Creemos tambien de interés investigar los parientes de niños afectados por esta deficiencia, ya que se describe una mayor incidencia familiar de esta alteración $(1,4)$. En nutestra serie, esta investigación se efectuó en 3 casos, siendo positiva en 1 de ellos (MMZ, Tabla No 3; presenta 1 hermano con IgA persistentemente disminuida y una prima con deficiencia parcial e infecciones a repetición, VSZ, Tabla No 2).

Respecto al tratamiento, es bien conocida la ineficacia de la gamaglobulina, e incluso sus riesgos, ya que posee sólo una mínima fracción de $\mathrm{IgA}$, la cual puede desencadenar fenómenos de hipersensibilidad por anticuerpos anti-IgA. Se usó en 2 casos de nuestra casuística, ambos con deficiencia combinada de la inmunidad humoral (IgG e IgA disminuidas). $\sin$ presentar problemas. En todos los otros casos, el tratamiento se limitó al de las enfermedades intercurrentes. En el caso de los niños con infecciones a repetición en Ios 2 primeros años de vida, se debe tratar de evitar el uso precoz e indiscriminado de antibib́ticos, logrando de esta manera que la infeccion por sf misma se convierta en el meior estímulo para el desarrollo del sistema inmune.

\section{SUMMARY}

18 children with IgA deficiency are presen- ted. The clinical and immunological findings allow to group them into three cathegories:

1) Selective IgA deficiency with IgA levels below $5 \mathrm{mg} \% .3$ out of 4 patients belonging to this group had a rheumatoid arthritis, and one a nephroticc syndrome. 2 out of the 3 children with arthritis had demonstrable autoimmune phenomena. The evolution of the arthritis was benign in the three cases. These children did not have an important tendency to present respiratory and digestive infections.

2) Partial IgA deficiencies with levels of IgA respectedly below the normal value, but over $5 \mathrm{mg} \%$ : These were usually children under 2 years of age, with frecuent infections of the upper resperatory tract. ( 7 out of 10). The older children belonging to this group were diagnosed after 8 years of age, all having a theumatoid arthritis with detectable autoinmune phenomena, and also with benign evolution.

3) IgA deficiency asociated with other immunodeficiencies: partial IgG-deficiency ( 2 cases) or cellular deficiencies as detected by negative skin test for PPD, monilia and DNCB. This group presented very frecuent respiratory and digestive infections, and, in one case, acute lynfoblastic leucemia and chronic active hepatitis.

\section{REFERENCIAS}

J. Stiehm, E.R. and Fulginiti, V. A.: Inrmunologic disorders in infants and children W.B. Saunders Company, 1973.

2. Tomasi, Th. B.: N. Engl. J. Med. 287: 500, 1972.

3. Primary Immunodeficiencies. Bull Wld. Org. 45: 125, 1971.

4. Grundbacher, F.J.: 1. Med. Genet. 9: 344, 1973.

5. Sörensen, R.: Rev. Chil. Ped. 44: 225, 1973.

6. Claman, H.N. et al.; J. Lab. Clin. Med. 75: 307, 1970.

7. Buser, F. et al.: J. Pediat. 72: 29, 1968.

8. Amman, A.J. and Hong, R.: J. Lab. and Clin. Med. 74: 846, 1969.

9. Taylor, B. et al.: Lancet 2: 111, 1973.

10. Savilahti, E. et al.: Arch. Dis. Child. 46: 665 , 1971. 